\title{
Development of a new method for assessing the cardiac baroreflex: response to downward tilting in patients with diabetes mellitus
}

M Nakagawa, N Takahashi, T Ooie, K Yufu, M Hara, M Watanabe, S Nobe, H Yonemochi, I Katsuragi, T Okeda, T Sakata, T Saikawa

\begin{abstract}
Objective-To investigate the clinical value of a new non-invasive method for assessing baroreflex sensitivity using downward tilting.

Patients-34 patients with diabetes mellitus, mean (SD) age, 53.6 (11.8) years.

Design-Arterial blood pressure and ECG were recorded simultaneously while the patients were on a tilt table. After 20 minutes at a $70^{\circ}$ upright tilt, the patients were returned to the supine position at a speed of $3.2 \%$ (downward tilting baroreflex sensitivity test, DT-BRS). A beat to beat systolic blood pressure increase associated with a corresponding lengthening of the RR interval was noted during downward tilting. Baroreflex sensitivity was also assessed using the conventional method of an intravenous injection of phenylephrine (Phe-BRS). Heart rate variability was analysed during rest and tilting.

Results-The slope of the regression line for systolic blood pressure $v$ RR interval during downward tilting was highly correlated with Phe-BRS $(r=0.83, \mathrm{p}<0.0001)$. Both DT-BRS and PheBRS were correlated with the high frequency $(\mathrm{HF})$ component of resting heart rate variability $(\mathrm{p}<0.005)$ and with the ratio of the low frequency to the high frequency component $(\mathrm{LF} / \mathrm{HF})$ during upright tilting $(\mathrm{p}<0.005)$. DT-BRS and Phe-BRS were also correlated with the difference between rest and tilting values of $\mathrm{HF}$ and $\mathrm{LF} / \mathrm{HF}(\mathrm{p}<0.005)$.

Conclusions-DT-BRS provides a physiological, non-invasive method for determining baroreflex sensitivity and may be a useful index of reflex cardiac vagal and sympathetic function in patients with diabetes mellitus.

(Heart 2001;86:643-648)
\end{abstract}

Keywords: baroreflex sensitivity; downward tilting; heart rate variability; diabetes

Baroreflex sensitivity is a marker of the ability to augment vagal activity. Previous experimental and clinical studies have shown that reduced baroreflex sensitivity is associated with an increased risk of lethal ventricular arrhythmias following myocardial infarction. ${ }^{1-5}$ The method most extensively described for assessing baroreflex sensitivity involves bolus intravenous injections of phenylephrine. ${ }^{6}$ Several non-invasive methods have been proposed for quantifying baroreflex sensitivity, such as the spectral $^{78}$ and sequence methods. ${ }^{910}$ We have recently developed a new non-invasive method to evaluate the baroreflex, using downward tilting. In our previous study conducted in healthy volunteers, ${ }^{11}$ we showed a strong correlation between systolic blood pressure increase and corresponding RR interval lengthening during downward tilting (downward tilt baroreflex sensitivity test, DT-BRS), which yielded DTBRS values that correlated well with those obtained by the phenylephrine method (PheBRS). The DT-BRS value also correlated significantly with the high frequency component of basal heart rate variability. We suggested that assessment of the baroreflex response to downward tilting could serve as a non-invasive index of reflex cardiac vagal function. However, because we tested a relatively small homogeneous group of young healthy male subjects, it was not clear whether DT-BRS would be a reliable method for assessing autonomic function in the clinical setting-for example, in patients with heart failure or diabetes mellitus.

Autonomic neuropathy is a common complication of diabetes mellitus and is associated with increased mortality. ${ }^{12}{ }^{13}$ Several studies have shown that baroreflex sensitivity is impaired in diabetes, in both the experimental ${ }^{14-16}$ and the clinical setting. ${ }^{17-20}$ Our main purpose in the present study was to assess the baroreflex response during downward tilting in patients with diabetes mellitus, to confirm whether this new method is a clinically useful test in patients whose reflex cardiac vagal activity requires clinical assessment. It has been shown that the parasympathetic nervous system mediates heart rate variability predominantly in the supine position, whereas during orthostatic positioning (such as upright tilting) the sympathetic nervous system predominates. ${ }^{21}$ We therefore also evaluated the contribution of the vagal and sympathetic nervous systems to the impaired control of baroreflexes in diabetes by comparing heart rate variability at rest and during head up tilt.

\section{Methods}

PATIENT POPULATION

We studied 34 patients with diabetes mellitus (12 men, 22 women) aged $26-71$ years (mean (SD) age, 53.6 (11.8) years). Four patients had type 1 diabetes and the remaining 30 patients 
had type 2. They had a mean disease duration of 8.2 (7.1) years. Patients with atrial fibrillation, frequent atrial or ventricular arrhythmias, or atrioventricular block were excluded, as were those with clinical evidence of heart failure or renal failure, hypertension, peripheral vascular disease, or a history of myocardial infarction and cerebrovascular disease. Nineteen patients were being treated with insulin $(n=11)$ or oral antidiabetic agents $(n=8)$ at the time of the study. None of the patients was taking any drug treatment known to affect the autonomic nervous system, such as $\beta$ blockers.

Written informed consent was obtained from all the subjects and the study was approved by our institution's review board.

STUDY PROTOCOL

Head up tilt testing was done in the morning (9 am to $11 \mathrm{am}$ ), after an overnight fast. Each patient was placed in the supine position on an electric tilt table with a footboard in a comfortable environment, and was instructed to breathe regularly at a rate of about 15 breaths/ min. Arterial blood pressure was recorded non-invasively using tonometry (Jentow-7700, Nihon Colin, Komaki, Japan). The tonometric sensor was attached over the left radial artery. To keep the level of the heart and the tonometric sensor equal during tilting, the left arm was held horizontally on a plate attached to the table. The accuracy of continuous blood pressure monitoring using this system has been demonstrated previously. ${ }^{22}$ Arterial blood pressure and the standard 12 lead ECG were monitored simultaneously, and data were stored in a PCM data recorder (RD-200T, TEAC, Tokyo, Japan) for subsequent analysis. A Holter ECG (model 459, Del Mar Avionics, Irvine, California, USA) was also recorded throughout the procedure for analysis of heart rate variability. A venous catheter was placed in the right median cephalic vein for intravenous phenylephrine injection.

After 30 minutes of equilibration, passive upward tilting from $0^{\circ}$ to $70^{\circ}$ was performed and maintained for 20 minutes. Thereafter, the table was returned to the horizontal position at a speed of $3.2 \%$. Fifteen minutes after completion of downward tilting, at which time the heart rate and blood pressure had completely returned to steady state levels, the baroreflex sensitivity was assessed by the conventional phenylephrine method (PheBRS). ${ }^{6}$ Phenylephrine $(1-3 \mu \mathrm{g} / \mathrm{kg})$ was injected over five seconds to obtain a $15-40 \mathrm{~mm} \mathrm{Hg}$ increase in systolic blood pressure. Baroreflex sensitivity was then calculated as the slope of

Table 1 Heart rate, blood pressure, and heart rate variability at rest and during tilt

\begin{tabular}{lcc}
\hline Variable & \multicolumn{1}{c}{ Rest } & \multicolumn{1}{c}{ Tilt } \\
\hline Heart rate (beats/min) & $68.1(12.1)$ & $82.2(11.7)^{\star \star}$ \\
Systolic blood pressure (mm Hg) & $124.1(17.7)$ & $106.9(17.2)^{\star \star}$ \\
Diastolic blood pressure (mm Hg) & $65.7(10.2)$ & $64.7(9.0)$ \\
LF component & $3.97(1.10)$ & $3.49(1.22)^{\star}$ \\
HF component & $3.89(1.19)$ & $2.52(0.96)^{\star \star}$ \\
LF/HF & $1.36(0.91)$ & $3.71(2.77)^{\star \star}$ \\
\hline
\end{tabular}

Data are mean $(\mathrm{SD})$.

${ }^{\star} \mathrm{p}<0.001,{ }^{\star \star} \mathrm{p}<0.0001 v$ rest.

LF and HF component, low and high frequency components of heart rate variability. Measured values of LF and HF were transformed by a natural logarithm. the linear regression line relating systolic blood pressure to the RR interval. Regression lines with a significant correlation coefficient $(r)$ greater than 0.8 were accepted for subsequent analysis. Injection of phenylephrine was repeated twice and the mean of the two slopes was taken as the baroreflex sensitivity value.

Baroreflex sensitivity measured by the downward tilting method was calculated as the slope of the linear regression line relating systolic blood pressure to RR interval during downward tilting. Regression lines with $r>0.8$ were accepted for analysis. Patients in whom tilting was discontinued because of the development of pronounced hypotension or bradycardia were excluded from the study.

To assess the reproducibility of the DT-BRS measurements, the same protocols for head up tilt were repeated 15 minutes after the first procedure in 10 randomly selected patients.

HEART RATE VARIABILITY ANALYSIS

Heart rate variability was analysed over a 300 second interval on Holter ECG records made during supine rest and after 15 minutes of head up tilt. The power spectrum of the RR intervals was computed by fast Fourier transform and expressed as the area under the power spectrum curve. We calculated the power of two spectral bands - the low frequency component (LF, 0.04-0.15 Hz) and the high frequency component $(\mathrm{HF}, 0.15-0.40 \mathrm{~Hz})-$ and computed the ratio of $\mathrm{LF}$ to $\mathrm{HF}$ (LF/HF). The difference between rest and tilting values of LF $(\Delta \mathrm{LF}), \mathrm{HF}(\Delta \mathrm{HF})$, and $\mathrm{LF} / \mathrm{HF}(\Delta \mathrm{LF} /$ $\mathrm{HF}$ ) were computed. Measured values of LF and $\mathrm{HF}$ were transformed by a natural logarithm because their distribution was skewed.

STATISTICAL ANALYSIS

Data are presented as mean (SD). Student's $t$ test for paired observations was used to evaluate changes produced by the tilt manoeuvre. The correlation between two variables was evaluated by Pearson's correlation coefficient. Correlation coefficients $(r)$ were tested for significance by $t$ tests. The method of Bland and Altman $^{23}$ was used to determine limits of agreement between Phe-BRS and DT-BRS. Limits of agreement were defined as the mean difference in baroreflex sensitivity \pm 2 SD. A probability value of $\mathrm{p}<0.05$ was considered significant.

\section{Results}

CHANGES IN BLOOD PRESSURE AND HEART RATE VARIABILITY DURING HEAD UP TILTING

Table 1 shows the effects of the head up tilt manoeuvre on heart rate, blood pressure, and the indices of heart rate variability. During tilt, heart rate and the LF:HF ratio of heart rate variability increased significantly, although systolic blood pressure, LF, and HF were all reduced. There was no significant change in diastolic blood pressure on tilting. 
CHANGES IN BLOOD PRESSURE AND RR INTERVAL DURING DOWNWARD TILTING

During downward tilting, a rise in systolic blood pressure (106.9 (17.2) to 130.8 (20.0) $\mathrm{mm} \mathrm{Hg}$ ) and a lengthening of the RR interval (708.9 (94.3) to $846.6(129.3) \mathrm{ms})$ occurred in all 34 patients. There was a significant linear correlation between systolic blood pressure increase and the corresponding $R R$ interval lengthening in each patient, as illustrated for a 26 year old female patient in fig 1 . In this patient, initiation of downward tilting induced a beat to beat increase in systolic blood pressure that reached a maximum approximately 20 seconds after the start of the downward tilting (bottom tracing in fig 1A). This increase in systolic blood pressure was accompanied by an increase in the corresponding RR interval (top and middle tracings in fig $1 \mathrm{~A}$ ). Plotting the corresponding RR interval against systolic blood pressure in this subject showed a strong correlation $(r=0.91, \mathrm{p}<0.0001$; fig $1 \mathrm{~B})$, yielding a DT-BRS value of $6.0 \mathrm{~ms} /$ $\mathrm{mm} \mathrm{Hg}$.

The values of DT-BRS obtained in this group of patients varied widely, from 0.01 to $12.7 \mathrm{~ms} / \mathrm{mm} \mathrm{Hg}$ (mean (SD) 4.8 (3.4) ms/ $\mathrm{mm} \mathrm{Hg})$.
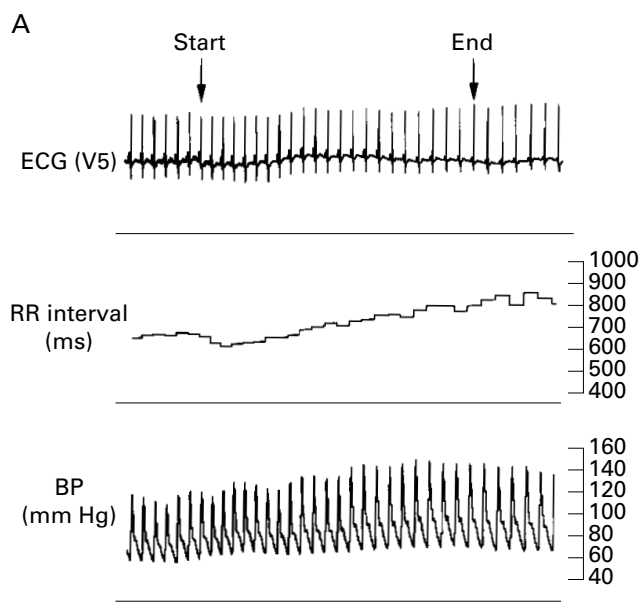

$\overline{10 \text { seconds }}$

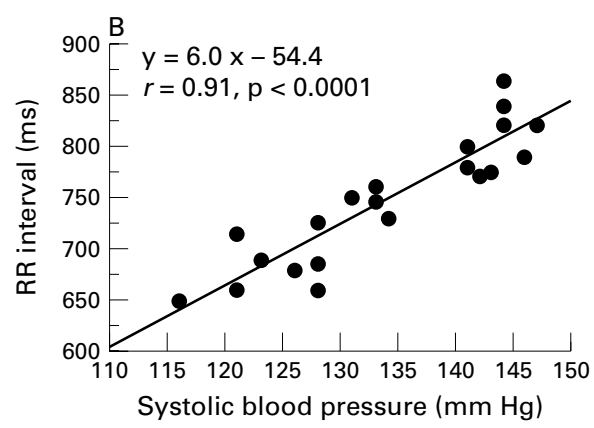

Figure 1 Relation between changes in systolic blood pressure increase and corresponding RR interval lengthening in a 26 year old female patient during downward tilting. (A) Top and middle panels: ECG tracing of lead V5 and computed RR interval. Bottom panel: arterial blood pressure (BP) recorded by tonometry. Arrows indicate the start and end of downward tilting. (B) Plotting of corresponding RR interval against systolic blood pressure in this patient showed a strong correlation $(r=0.91, p<$ 0.0001 ), yielding a baroreflex sensitivity value of 6.0 $\mathrm{ms} / \mathrm{mm} \mathrm{Hg}$.
The reproducibility of the DT-BRS value was assessed as the standard deviation of the differences between the first and second determination and was expressed as a percentage of the average value. Reproducibility was $7.1 \%$ and the correlation coefficient was 0.97 $(\mathrm{p}<0.0001)$.

COMPARISON OF DT-BRS WITH PHE-BRS

In all patients, intravenous injection of phenylephrine caused an increase in systolic blood pressure (from 124.1 (17.1) to 148.3 (15.4) $\mathrm{mm} \mathrm{Hg}$ ), which correlated significantly with lengthening of the corresponding RR interval (from 863.7 (125.0) to 1048.7 (193.0) $\mathrm{ms}$ ). The mean value of the rise in systolic blood pressure during Phe-BRS was similar to that during DT-BRS (24.2 (10.9) $v$ 23.9 (9.1) $\mathrm{mm} \mathrm{Hg}$ ); however, the mean value of $\mathrm{RR}$ interval lengthening in Phe-BRS was greater than in DT-BRS $(185.0(110.2) v$ 137.7 (91.4) $\mathrm{ms}, \mathrm{p}=0.0003$ ).

The values of Phe-BRS also varied widely in our population sample, from 0.01 to $25.0 \mathrm{~ms} /$ $\mathrm{mm} \mathrm{Hg}$ (mean (SD), 7.4 (6.4) $\mathrm{ms} / \mathrm{mm} \mathrm{Hg}$ ).

Three representative examples of the correlation between systolic blood pressure increase and corresponding $\mathrm{RR}$ interval lengthening during the downward tilting and phenylephrine methods are shown in fig 2. A 27 year old patient had a normal Phe-BRS (15.5 ms/ $\mathrm{mm} \mathrm{Hg}$ ) and her DT-BRS value was also high $(9.3 \mathrm{~ms} / \mathrm{mm} \mathrm{Hg}$ ) (fig $2 \mathrm{~A})$. In contrast, PheBRS obtained from a patient aged 63 years fell to $3.6 \mathrm{~ms} / \mathrm{mm} \mathrm{Hg}$ and her DT-BRS value was virtually the same (fig $2 \mathrm{~B}$ ). Another patient, a 61 year old woman, showed a major reduction in both Phe-BRS $(1.1 \mathrm{~ms} / \mathrm{mm} \mathrm{Hg})$ and DTBRS (0.8 $\mathrm{ms} / \mathrm{mm} \mathrm{Hg}$ ) (fig $2 \mathrm{C}$ ). We evaluated the relation between the values of DT-BRS and Phe-BRS in all 34 patients (fig 3). DT-BRS was well correlated with Phe-BRS $(r=0.83$, $\mathrm{p}<0.0001)$, though the mean DT-BRS value was significantly lower than the mean Phe-BRS value, at 4.8 (3.4) $v 7.4 \quad(6.4) \mathrm{ms} / \mathrm{mm} \mathrm{Hg}$ $(\mathrm{p}<0.0005)$. The mean difference in baroreflex sensitivity as determined by the two methods was $2.7 \mathrm{~ms} / \mathrm{mm} \mathrm{Hg}$, and the limits of agreement were -5.4 to $10.8 \mathrm{~ms} / \mathrm{mm} \mathrm{Hg}$.

COMPARISON OF BAROREFLEX SENSITIVITY WITH HEART RATE VARIABILITY AT REST AND DURING TILT

In all patients studied, DT-BRS was correlated significantly with the power of the LF and HF components of heart rate variability at rest and with the LF and LF/HF ratio during tilt, but not with the $\mathrm{HF}$ during tilt or the $\mathrm{LF} / \mathrm{HF}$ ratio at rest (table 2). DT-BRS was also correlated with $\Delta \mathrm{LF}, \Delta \mathrm{HF}$, and $\Delta \mathrm{LF} / \mathrm{HF}$. Phe-BRS was significantly correlated with the indices of heart rate variability, and the correlation coefficients were almost equivalent to those obtained with DT-BRS.

\section{Discussion}

In this study we assessed cardiac baroreceptor reflex sensitivity using a downward tilting method and attempted to explore the relation between this method and the conventional 

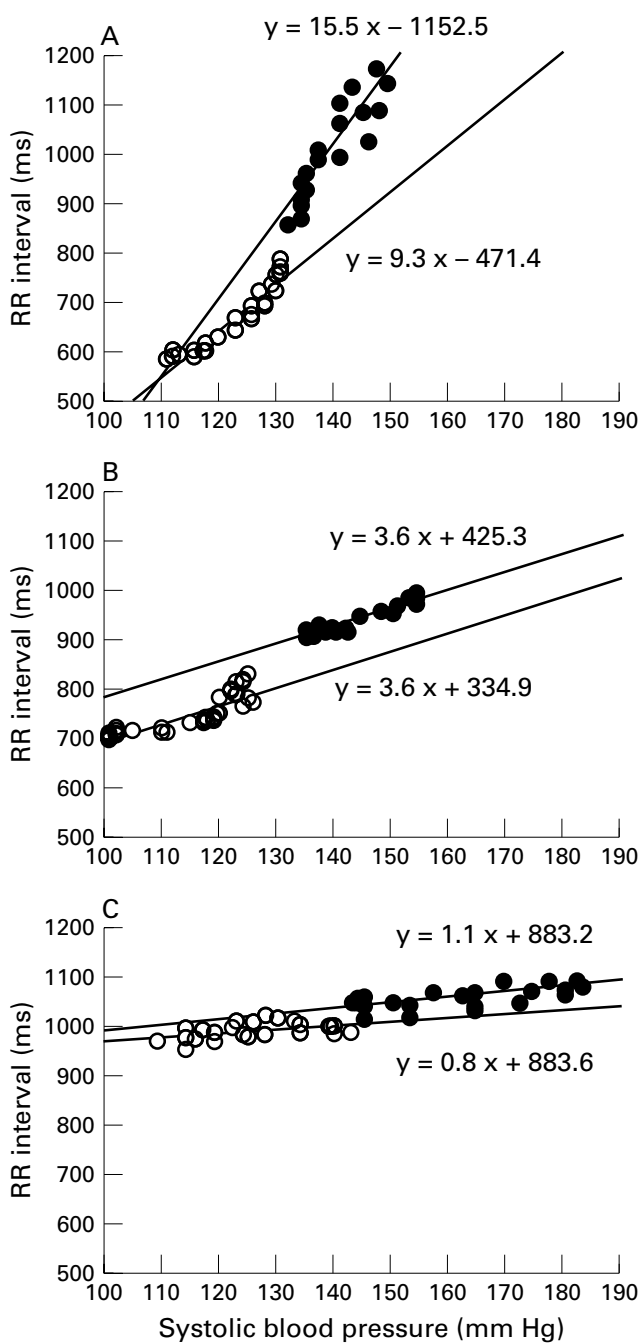

Figure 2 Relation between systolic blood pressure increase and corresponding $R R$ interval lengthening during downward tilting (empty circles) and phenylephrine injection (filled circles) in three representative examples of the patients studied. (A) A patient aged 27 years showing a normal Phe-BRS value $(15.5 \mathrm{~ms} / \mathrm{mm} \mathrm{Hg})$ and a slightly lower DT-BRS value $(9.3 \mathrm{~ms} / \mathrm{mm} \mathrm{Hg})$. (B) Phe-BRS obtained a patient aged 63 years was reduced to $3.6 \mathrm{~ms} / \mathrm{mm}$ $\mathrm{Hg}$ and the DT-BRS value was virtually the same. (C) $A$ 61 year old female patient with a major reduction in both Phe-BRS (1.1 ms $/ \mathrm{mm} \mathrm{Hg}$ ) and DT-BRS $(0.8 \mathrm{~ms} / \mathrm{mm}$ $\mathrm{Hg}$ ).

phenylephrine method in patients with diabetes mellitus. The major finding was a significant correlation between the rise in systolic blood pressure and lengthening of the RR interval during downward tilting. We also showed that DT-BRS values were well correlated with Phe-BRS values and with heart rate variability.

ASSESSMENT OF BAROREFLEX SENSITIVITY

The ability to augment vagal activity can be determined by baroreflex sensitivity. Although several quantitative approaches have been developed for evaluating baroreflex sensitivity, the method used most extensively involves a bolus injection of the pressor drug phenylephrine. ${ }^{1-6}$ The disadvantage of the phenylephrine method is that it involves the intravenous administration of a pressor agent

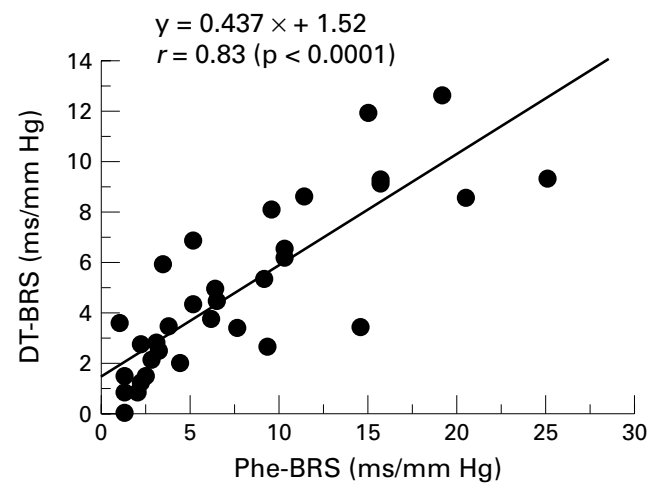

Figure 3 Correlation between baroreflex sensitivity measured by the downward tilting method (DT-BRS) and by the phenylephrine method (Phe-BRS) in 34 patients with diabetes mellitus. DT-BRS correlated significantly with Phe-BRS.

to raise the blood pressure, which artificially disrupts physiological cardiovascular homeostasis. In addition, as systolic blood pressure sometimes increases rather greatly after phenylephrine administration (as shown in fig $2 \mathrm{C}$ ), this method may not be feasible in cases where there is pronounced hypertension or a significant risk of cardiovascular events. Recently, several non-invasive methods of assessing baroreflex sensitivity have been proposed, including spectral analysis ${ }^{7}$ and sequential beat by beat analysis of spontaneous oscillations of blood pressure and RR interval. ${ }^{9}{ }^{10}$ 17-20 Both spectral analysis and the sequential method can detect the minimal recruitment of cardiac vagal efferents by natural pressure variations. However, as those methods require special computer analysis techniques they have not been widely used in the clinical setting.

Recently, we proposed a new non-invasive method to assess baroreflex sensitivity using downward tilting. ${ }^{11}$ We assessed the relation between changes in systolic blood pressure and $\mathrm{RR}$ interval during downward tilting in healthy subjects and found a significant correlation between these variables. We called this noninvasive approach DT-BRS. DT-BRS also correlated significantly with baroreflex sensitivity measurements obtained by the phenylephrine method. In our previous study, however, only a small homogeneous group of healthy young

Table 2 Correlation of baroreflex sensitivity with heart rate variability

\begin{tabular}{lll}
\hline Variability index & $D T-B R S$ ( $r$ value) & Phe-BRS ( $r$ value) \\
\hline LF (rest) & $0.656^{\star \star \star}$ & $0.685^{\star \star \star}$ \\
HF (rest) & $0.563^{\star \star}$ & $0.524^{\star \star}$ \\
LF/HF (rest) & 0.078 & 0.201 \\
LF (tilt) & $0.583^{\star \star}$ & $0.565^{\star \star}$ \\
HF (tilt) & 0.211 & 0.188 \\
LF/HF (tilt) & $0.541^{\star \star}$ & $0.516^{\star \star}$ \\
$\Delta \mathrm{LF}$ & $0.550^{\star}$ & $0.745^{\star}$ \\
$\Delta \mathrm{HF}$ & $0.574^{\star \star}$ & $0.532^{\star \star}$ \\
$\Delta \mathrm{LF} / \mathrm{HF}$ & $0.567^{\star \star}$ & $0.495^{\star}$ \\
\hline
\end{tabular}

Values are correlation coefficients of the linear regression analysis between downward tilt baroreflex sensitivity measurement (DT-BRS) and conventional phenylephrine assessment (PheBRS) versus indices of heart rate variability.

${ }^{\star} \mathrm{p}<0.01,{ }^{\star \star} \mathrm{p}<0.005,{ }^{\star \star \star} \mathrm{p}<0.0001$.

$\mathrm{LF}$ and $\mathrm{HF}$, low and high frequency components of heart rate variability; $\Delta \mathrm{LF}, \Delta \mathrm{HF}$, and $\Delta \mathrm{LF} / \mathrm{HF}$, the difference between rest and tilt values of $\mathrm{LF}, \mathrm{HF}$, and $\mathrm{LF} / \mathrm{HF}$, respectively. Measured values of LF and HF were transformed by a natural logarithm. 
subjects was tested. ${ }^{11}$ Baroreflex control in diseased patients - such as those with hypertension, heart disease, or diabetes-differs from that in healthy subjects. Patients with diabetes mellitus often have autonomic disturbances with impaired baroreflex sensitivity. ${ }^{17-20}$ We found a wider distribution of DT-BRS values in our diabetic patients than in the healthy subjects. ${ }^{11}$

COMPARISON BETWEEN DT-BRS AND PHE-BRS DT-BRS values were significantly correlated with Phe-BRS values in patients with diabetes mellitus in the present study, as in our normal controls. ${ }^{11}$ This suggests that DT-BRS provides an assessment of reflex cardiac vagal function comparable with that obtained by the phenylephrine method, though the values obtained with the downward tilting method were lower than with the phenylephrine method.

There are several possible reasons for the difference between the DT-BRS and Phe-BRS values.

First, with the phenylephrine method a vasoconstriction induced rise in blood pressure stimulates arterial baroreceptors, causing activation of vagal efferents; this results in lengthening of the RR interval. Conversely, the rise in blood pressure that occurs in response to downward tilting is thought mainly to reflect the increased venous return, which is followed by an increase in cardiac output. This must involve activation of cardiopulmonary baroreceptors in addition to arterial baroreceptors. Thus DT-BRS may assess both cardiopulmonary and arterial baroreceptor sensitivity.

Second, although the gain in systolic blood pressure during in downward tilting was similar to that with phenylephrine administration (mean (SD), 23.9 (9.1) v 24.2 (10.9) $\mathrm{mm} \mathrm{Hg}$ ), the level of systolic blood pressure achieved with these two methods was different, in that blood pressure at the start and end of downward tilting was lower than in the phenylephrine method, as shown in fig 2. Baroreflex control in the physiological state in human subjects is not static, and dynamic resetting of the threshold occurs depending on the level of arterial blood pressure. ${ }^{24}$ While the gain of the phenylephrine induced baroreflex is an index of maximum recruitment of cardiac vagal efferents during extremes of parasympathetic engagement caused by artificially induced pressure increases, the baroreflex obtained by downward tilting assesses a more physiological level of recruitment of cardiac vagal efferent neurones.

Third, while the phenylephrine method is performed in a resting supine position with high vagal tone and sympathetic inhibition, downward tilting was begun from a $70^{\circ}$ upright posture in which sympathetic activity substantially increased. ${ }^{21}$ In patients with heart disease, such as heart failure, sympathetic tone is substantially increased and this plays an important role in triggering lethal arrhythmias. Accordingly, baroreflex sensitivity assessment during downward tilting may be more useful than the phenylephrine method under such conditions, as it evaluates the baroreflex response in the setting of increased sympathetic tone.

COMPARISON WITH HEART RATE VARIABILITY Cardiac autonomic tone can be assessed reliably by spectral analysis of heart rate variability. HF power provides a marker of vagal tone, and the $\mathrm{LF} / \mathrm{HF}$ ratio is an index of sympathetic activity. ${ }^{25}$ The LF component is modulated by baroreflex perturbation. ${ }^{26}$ In the present study, DT-BRS and Phe-BRS correlated significantly with the power of $\mathrm{HF}$ at rest. However, in these correlations, the correlation coefficient was not very high ( $r=0.52$ to 0.56 ). Studies comparing baroreflex sensitivity with heart rate variability in postinfarction patients showed only a moderate correlation between the two methods. ${ }^{27}$ Our results are consistent with these results. Although the HF components of heart rate variability and baroreflex sensitivity are both indices of vagal activity, they reflect different physiological aspects. The former is a marker of tonic vagal outflow, whereas the latter is a marker of reflex vagal outflow.

Both DT-BRS and Phe-BRS were correlated positively with the $\mathrm{LF} / \mathrm{HF}$ ratio during tilt. Head up tilt induces a physiological increase in sympathetic activity. In this study, tilting caused a decrease in $\mathrm{HF}$ and an increase in $\mathrm{LF} / \mathrm{HF}$, suggesting parasympathetic withdrawal and an enhanced sympathetic tone, which are consistent with previous studies conducted in healthy controls. ${ }^{21} 28$ Thus the analysis of heart rate variability during tilt, especially the change of LF/HF from the supine position $(\Delta \mathrm{LF} / \mathrm{HF})$, may be useful in the assessment of cardiac reflex sympathetic function. The arterial baroreflex influences both the sympathetic and parasympathetic limbs of the autonomic nervous system and cardiovascular function. Diabetic patients with autonomic dysfunction have reduced vagal tone combined with a reduced capacity to activate sympathetic tone. ${ }^{29}$ The relation of DT-BRS to LF/HF during tilt and to $\Delta \mathrm{LF} / \mathrm{HF}$ shown in this study suggests that DT-BRS could be a useful tool for assessing reflex cardiac vagal and sympathetic activity in patients with diabetes mellitus.

\section{LIMITATIONS}

Our study has several limitations. First, an electrical tilt table is necessary for DT-BRS, and this may not be generally available in clinical departments. Second, while $70^{\circ}$ passive upright tilting was maintained for 20 minutes in the present study, the angle, duration of upright tilt, and the speed of downward tilt should be varied to determine the optimal conditions for baroreflex sensitivity assessment. Third, although the reproducibility of the DT-BRS values was very good, in clinically unstable patients baroreflex sensitivity determination at rest - such as spectral analysis or the sequential method-might be more suitable than DT-BRS. Finally, prospective evaluation of the prognostic significance of the results will be necessary to confirm the clinical value of DT-BRS in a larger and more heterogeneous group of patients. 
CONCLUSIONS

We evaluated a new method for assessing baroreflex sensitivity, DT-BRS. We confirmed that this provided a non-invasive and quantitative means of assessing cardiac vagal and sympathetic function in patients with diabetes mellitus.

We gratefully acknowledge the technical assistance provided by Emi Noguchi BS.

1 La Rovere MT, Specchia G, Mortara A, et al. Baroreflex sensitivity, clinical correlates, and cardiovascular mortality among patients with a first myocardial infarction. A prospective study. Circulation 1988;78:816-24.

2 Farrell TG, Odemuyiwa O, Bashir Y, et al. Prognostic value of baroreflex sensitivity testing after acute myocardial of baroreflex sensitivity testing after

3 Hohnloser SH, Klingenheben T, van de Loo A, et al. Reflex versus tonic vagal activity as a prognostic parameter in patients with sustained ventricular tachycardia or ventricuar fibrillation. Circulation 1994;89:1068-73.

4 De Ferrari GM, Landolina M, Mantica M, et al. Baroreflex sensitivity, but not heart rate variability, is reduced in patients with life-threatening ventricular arrhythmias long after myocardial infarction. Am Heart 7 1995;130:473-80.

5 La Rovere MT, Bigger JT, Marcus FI, et al. Baroreflex sensitivity and heart-rate variability in prediction of total cardiac mortality after myocardial infarction. Lancet 1998 ; 351:478-84.

6 Smyth HS, Sleight P, Pickering GW. Reflex regulation of arterial pressure during sleep in man: a quantitative method of assessing baroreflex sensitivity. Circ Res 1969;24: 109-22.

7 Robbe HW, Mulder LJ, Ruddel H, et al. Assessment of baroreceptor reflex sensitivity by means of spectral analysis. bypertension 1987;10:538-43.

8 Pagani M, Somers V, Furlan R, et al. Changes in autonomic regulation induced by physical training in mild hypertension. Hypertension 1988;12:600-10.

9 Parati G, Di Rienzo M, Bertinieri G, et al. Evaluation of the baroreceptor-heart rate reflex by 24 -hour intra-arteria blood pressure monitoring in humans. Hypertension 1988; 12:214-22.

10 Parlow JP, Viale JP, Hughson R, et al. Spontaneous cardiac baroreflex in humans: comparison with drug-induced responses. Hypertension 1995;25:1058-68.

11 Takahashi N, Nakagawa M, Saikawa T, et al. Noninvasive assessment of the cardiac baroreflex; response to downward tilting and comparison with the phenylephrine method. F Am Coll Cardiol 1999;34:211-15.

12 Ewing DJ, Campbell IM, Clarke BF. The natural history of diabetic autonomic neuropathy. $O \dot{f}$ Med 1980;49:95-108.
13 Rathmann W, Ziegler D, Jahnke M, et al. Mortality in diabetic patients with cardiovascular autonomic neudiabetic patients with cardiovascular
ropathy. Diabetic Med 1993;10:820-4.

14 McDowell TS, Hajduczok G, Abboud FM, et al. Baroreflex dysfunction in diabetes mellitus. II. Site of baroreflex impairment in diabetic rabbits. Am f Physiol 1994;266: H244-9.

15 Maeda CY, Fernandes TG, Timm HB, et al. Autonomic dysfunction in short-term experimental diabetes. Hypertension 1995;26:1100-4.

16 Miller AW, Sims JJ, Canavan A, et al. Impaired vagal reflex activity in insulin-resistant rats. $\mathcal{f}$ Cardiovasc Pharmacol 1999;35:698-702

17 Weston PJ, Panerai RB, McCullough A, et al. Assessment of baroreceptor-cardiac reflex sensitivity using time domain analysis in patients with IDDM and the relation to left ventricular mass index. Diabetologia 1996;39:1385-91.

18 Frattola A, Parati G, Gamba P, et al. Time and frequency domain estimates of spontaneous baroreflex sensitivity provide early detection of autonomic dysfunction in diabetes mellitus. Diabetologia 1997;40:1470-5.

19 Lengyel C, Török T, Várkonyi T, et al. Baroreflex sensitivity and heart rate variability in insulin-dependent diabetes with polyneuropathy. Lancet 1998;351:1436-7.

20 Ducher M, Thivolet C, Cerutti C, et al. Noninvasive exploration of cardiac autonomic neuropathy. Diabetes Care 1999;22:388-93.

21 Vybiral T, Bryg RJ, Maddens ME, et al. Effect of passive tilt on sympathetic and parasympathetic components of heart rate variability in normal subjects. Am f Cardiol 1989;63: $1117-20$.

22 Sato T, Nishinaga M, Kawamoto A, et al. Accuracy of a continuous blood pressure monitor based on arterial tonometry. Hypertension 1993;21:866-74.

23 Bland JM, Altman DG. Statistical methods for assessing agreement between two methods of clinical measurement. Lancet 1986;8:307-10

24 Head GA. Baroreflexes and cardiovascular regulation in hypertension. 7 Cardiovasc Pharmacol 1995;26:S7-16.

25 Malliani A, Pagani M, Lombardi F, et al. Cardiovascular neural regulation explored in the frequency domain. Circulation 1991;84:482-92.

26 Sleight P, La Rovere MT, Mortara A, et al. Physiology and pathophysiology of heart rate and blood pressure variability in humans: is power spectral analysis largely an index of baroreflex gain? Clin Sci 1995;88:103-9.

27 Bigger JT, La Rovere MT, Steinman RC, et al. Comparison of baroreflex sensitivity and heart period variability after myocardial infarction. $\mathcal{F}$ Am Coll Cardiol 1989;14:1511-18.

28 Nakagawa $M$, Takahashi $\mathrm{N}$, Iwao $\mathrm{T}$, et al. Evaluation of autonomic influence on QT dispersion using the head-up tilt test in healthy subjects. PACE 1999;22:1158-63.

29 Bellavere F, Balzani I, De Masi G, et al. Power spectral analysis of heart rate variations improves assessment of diabetic cardiac autonomic neuropathy. Diabetes 1992;41: $633-40$.

\section{Electronic pages}

\section{eHEART: www.heartjnl.com}

The following electronic only articles are published in conjunction with this issue of Heart (see also p 678).

\section{Community acquired staphylococcal pulmonary valve endocarditis in non-drug users: case report and review of the literature \\ F F Edmond, $S \mathcal{F}$ Eykyn, L D Smith}

Right sided endocarditis usually involves the tricuspid valve, predominantly in intravenous drug users. It is also occasionally acquired in hospital as a result of contaminated intravascular devices. Isolated infection of the pulmonary valve is rarely seen. A case of community acquired Staphylococcus aureus pulmonary valve endocarditis that caused diagnostic confusion is reported. This infection occurred in a patient with no history of intravenous drug abuse and a previously structurally normal heart.

(Heart 2001;86:e17) www.heartjnl.com/cgi/content/full/ $86 / 6 /$ e 17

\section{Acute profound thrombocytopenia following angioplasty: the dilemma in the management and a review of the literature \\ $S N$ Makoni}

Abciximab, heparin, and clopidogrel are often used together in the setting of coronary syndromes. These drugs are associated with thrombocytopenia and it is important to quickly discriminate the cause of this complication as it has implications for the management of thrombocytopenia and the coronary syndrome. This case highlights some of the dilemmas that may arise as no test can definitively identify the offending drug, and stopping these drugs can affect the outcome of the coronary event including stent thrombosis.

(Heart 2001;86:e18) www.heartjnl.com/cgi/content/full/ $86 / 6 / \mathrm{e} 18$ 